$\infty$
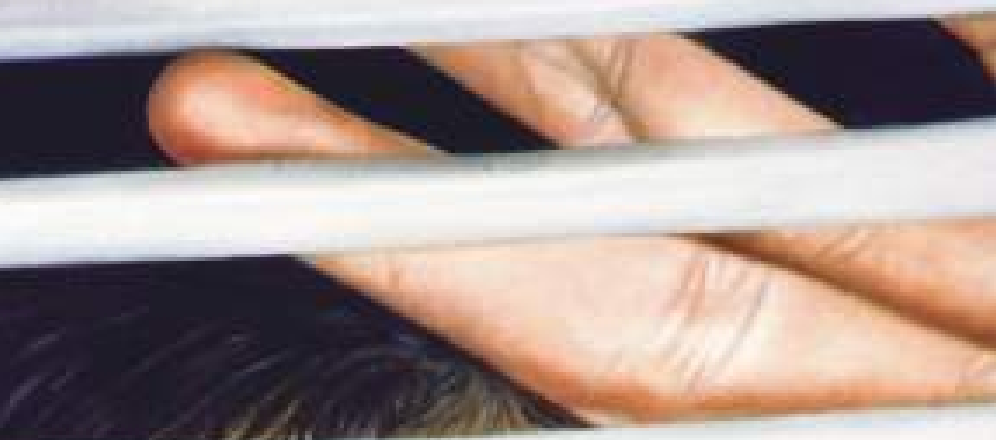

\title{
200
}

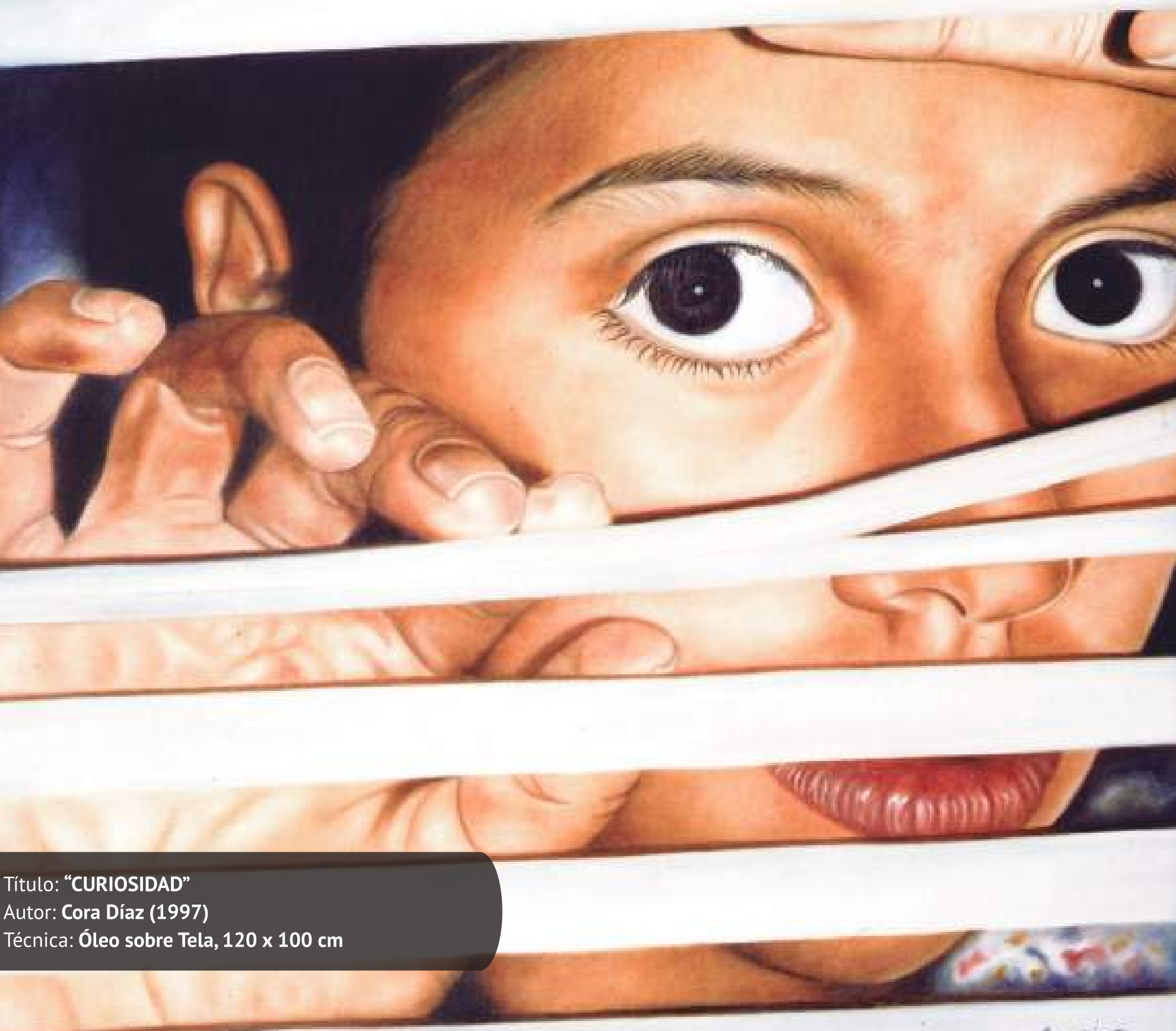




\section{CERTIFICACIÓN DE INGLÉS COMO LENGUA EXTRANJERA Y COMPRENSIÓN LECTORA}

CERTIFICATION OF ENGLISH AS A FOREIGN LANGUAGE AND READING COMPREHENSION

\section{AGLAE BEATRIZ FLORES LEOS MARÍA GUADALUPE RODRÍGUEZ BULNES}

\section{RESUMEN}

Este artículo presenta la deficiencia en la certificación del inglés como lengua extranjera en estudiantes del Instituto de Educación Superior en Estudios de la Salud (IESALUD) y se pretende preparar al estudiante con las habilidades lingüísticas adecuadas para que obtenga una certificación en el idioma inglés que avale su conocimiento. Para el proyecto se aplicó la metodología Investigación-Acción y el examen TOEFL PBT en 25 estudiantes para conocer las dificultades lingüísticas y, con base a ello, prepararlos para incrementar la habilidad que obtuvo menos puntaje. Los resultados obtenidos demostraron que la comprensión lectora fue la habilidad que más se les dificulta aprender a los estudiantes, por lo que se llevó a cabo una serie de actividades para incrementar esta habilidad.

PALABRAS CLAVE: INGLÉS, CERTIFICACIÓN, LECTURA, COMPRENSIÓN, ENSEÑANZA, MÉTODOS.

\begin{abstract}
This article presents the certification deficiency of English as a foreign language in students of the Instituto de Educación Superior en Estudios de la Salud (IESALUD); it is intended to prepare the student with the adequate linguistic skills to obtain a type of certification in the English language that supports their knowledge. For the project the action-research methodology was applied and as a tool the TOEFL PBT (Paper Based Test) was used in 25 students to know the linguistic difficulties and based on this, prepare them to increase the skill that obtained the lowest score. The results obtained test showed that reading comprehension was the most difficult skill to learn for the students of the Institute, so a series of activities was carried out to increase this ability.
\end{abstract}

KEYWORDS: ENGLISH, CERTIFICATION, READING, COMPREHENSION, TEACHING, METHODS. 
$\mathrm{E}$ I aprendizaje del inglés como lengua extranjera en nuestro país tiene un déficit importante en todos los niveles académicos. Uno de los inconvenientes es la falta de docentes capacitados adecuadamente para la impartición de un segundo idioma extranjero, en específico el inglés (Organización Mexicanos Primero, 2015). Los problemas se originan desde un sistema educativo inadecuado para la enseñanza, así como la poca importancia que se le da a la materia con base al currículo de los diferentes niveles educativos, desde la educación Básica hasta la Superior.

A pesar de la importancia que representa contar con el idioma inglés en el ámbito escolar, social y laboral, en México no se cuenta con un antecedente que promueva un cambio significativo para la impartición adecuada y eficiente de tal idioma. Actualmente, los estudiantes presentan dificultades a la hora de realizar una actividad 0 acudir a las tecnologías, dado que en la mayoría de los casos la información se encuentra en inglés. Esto provoca que el buscar información le sea difícil al estudiante (Ricoy y Álvarez, 2016).

El maestro juega un rol importante en el aprendizaje, ya que éste mismo se encarga de brindar las herramientas necesarias para que el alumno desarrolle sus habilidades. Se conoce que una gran cantidad de estudiantes no cuentan con algún tipo de certificación en el idioma inglés, por lo tanto, esto ocasiona una calidad baja en los estudiantes, afectando el desarrollo y el rendimiento académico de los mismos. Este estudio está centrado en conocer las debili- dades lingüísticas de los estudiantes del Instituto de Educación Superior en Estudios de la Salud (IESALUD) e identificar el nivel de inglés que poseen los estudiantes de acuerdo al Marco Común Europeo de Referencia para las Lenguas (MCER). Esto con la finalidad de preparar al estudiante con las habilidades lingüísticas adecuadas para lograr una certificación en el idioma inglés.

\section{PLANTEAMIENTO DEL PROBLEMA}

El principal problema que los estudiantes de inglés tienen es la deficiencia en el conocimiento del idioma. Actualmente, este problema está presente en los alumnos de Educación Superior y es uno de los factores que impide que se alcancen los principales objetivos de formar intelectualmente a estudiantes en una sociedad globalizada. El docente debe ser pieza clave en dichos objetivos para preparar a sus estudiantes en el ámbito escolar.

Es importante que los alumnos estén actualizados mediante pruebas, cursos y capacitaciones para conseguir una certificación que avale el conocimiento del idioma. Una vez obteniendo algún tipo de certificación, el alumno tendrá las herramientas adecuadas para sobresalir en su ámbito académico. Por consiguiente, al aprender el idioma inglés, le será más fácil interactuar con sus compañeros y de igual forma con el mismo profesor, el ambiente será dinámico, receptivo y atractivo logrando un mayor nivel en dicho idioma.

Hay estudiantes que tienen el conocimiento de un idioma extranjero, pero no cuentan con certificaciones que los respalden. Una vez que lo logren, podrán justificar sus conocimientos al mismo tiempo que se desempeñan mejor en la escuela y podrán aspirar a un mejor futuro laboral.

\section{MARCO TEÓRICO}

El Marco Común Europeo de Referencia para las Lenguas MCER (o sus siglas en inglés CEFR Common European Framework of Reference for Languages) sirve de referencia para describir el dominio de una lengua. En la Tabla 1 (pág. 49) se presentan los niveles que contempla el MCER.

\section{CERTIFICACIÓN DEL DOMINIO DEL IDIOMA EXTRANJERO}

El diccionario de la Lengua Española (2001) define que Certificar es Dar por Cierto, Afirmar o Asegurar Algo.
Uno de los inconvenientes es la falta de docentes capacitados adecuadamente para la impartición de un segundo idioma extranjero, en específico el inglés (Organización Mexicanos Primero, 2015) 
TABLA 1. Niveles Comunes de Referencia: Escala Global (Council of Europe, 2001)

\begin{tabular}{|c|c|c|}
\hline NIVEL MCER & USUARIO & DESCRIPCIÓN \\
\hline A1 & Básico & $\begin{array}{l}\text { Es capaz de comprender y utilizar expresiones cotidianas de uso muy frecuen- } \\
\text { te, puede presentarse a sí mismo y a otros, pedir y dar información personal } \\
\text { básica sobre su domicilio, sus pertenencias y las personas que conoce. }\end{array}$ \\
\hline A2 & Básico & $\begin{array}{l}\text { Es capaz de comprender frases y expresiones de uso frecuente, sabe comuni- } \\
\text { carse a la hora de llevar a cabo tareas simples y cotidianas y sabe describir en } \\
\text { términos sencillos aspectos de su pasado y su entorno. }\end{array}$ \\
\hline B1 & Independiente & $\begin{array}{l}\text { Es capaz de comprender los puntos principales de textos claros y en lengua } \\
\text { estándar si tratan sobre cuestiones que le son conocidas ya sea en situaciones } \\
\text { de trabajo, de estudio o de ocio, sabe desenvolverse en la mayor parte de las } \\
\text { situaciones, produce textos sencillos y justifica brevemente sus opiniones. }\end{array}$ \\
\hline B2 & Independiente & $\begin{array}{l}\text { Es capaz de entender las ideas principales de textos complejos que traten de } \\
\text { temas tanto concretos como abstractos, puede relacionarse con hablantes } \\
\text { nativos y puede producir textos claros y detallados. }\end{array}$ \\
\hline C1 & Competente & $\begin{array}{l}\text { Es capaz de comprender una amplia variedad de textos extensos y con cierto } \\
\text { nivel de exigencia así como reconocer en ellos sentidos implícitos, sabe } \\
\text { expresarse de forma fluida y puede hacer uso flexible del idioma. }\end{array}$ \\
\hline $\mathrm{C} 2$ & Competente & $\begin{array}{l}\text { Comprende prácticamente todo lo que oye o lee, sabe construir información y } \\
\text { puede expresarse con gran fluidez en un alto grado de precisión. }\end{array}$ \\
\hline
\end{tabular}

Con el certificado se permite evidenciar de una forma confiable y objetiva el manejo de un idioma determinado. Con este documento se evalúan, acreditan y certifican los conocimientos en materia de lenguas extranjeras. Hoy en día, existen diferentes exámenes, certificaciones o pruebas para cada tipo y necesidad del estudiante. Una de las organizaciones que elaboran este tipo de pruebas es el ETS (Educational Testing Service). La Universidad de Cambridge tambien realiza exámenes con ese mismo propósito.

\section{EXÁMENES REALIZADOS POR ELETS}

Uno de los exámenes realizados por la organización es el TOEFL (Test of English as a Foreign Language), prueba que se adapta al dominio del idioma inglés estadounidense y está orientada a hablantes no nativos de este idioma.

Otro examen es el EFSET (EF Standard English Test), test de inglés usado principalmente por adultos con fines de certificación profesional del inglés. Como es de libre acceso, las escuelas y las empresas también lo utilizan para medir el nivel de inglés de estos mismos.

Y como último ejemplo, tenemos el TOEIC (Test of English for International (Communication), examen orientado a empresas o profesionales que no tienen el idioma como lengua materna y está diseñado para evaluar los conocimientos de inglés en un entorno profesional (Educational Testing Service, 2017).

\section{EXÁMENES REALIZADOS POR LA UNIVERSIDAD DE CAMBRIDGE}

El KET (Key English Test) es un certificado que demuestra un nivel elemental del idioma inglés, es decir, el candidato puede comunicarse en situaciones sencillas. El PET (Preliminary English) es un certificado que respalda un nivel intermedio en el dominio del idioma inglés, aquellos que lo aprueban obtienen un certificado que no caduca. Y por último tenemos el CPE (Certificate of Proficiency in English), este examen es el más alto en cuanto a nivel de inglés se refiere, el candidato demuestra que domina el inglés a un nivel casi nativo (Education First, 2017). 


\section{COMPRENSIÓN LECTORA}

Johnston, Barnes y Desrochers (2008) definen la comprensión lectora como la construcción de una representación mental de la información textual y su interpretación. Jitendra y Gajria (2011) mencionan que la comprensión lectora es el método intencionado de extraer el significado del texto. La comprensión de textos se encuentra en todos los niveles académicos y se considera importante a la hora de aprender un idioma extranjero, ya que los alumnos se van familiarizando con el texto al mismo tiempo que van incrementan su vocabulario.

\section{MÉTODOS UTILIZADOS EN LA ENSEÑANZA DE UNA LENGUA EXTRANJERA}

Los métodos pueden servir como modelos de integración de la Teoría (Principios)y la Práctica (Técnicas).Su estudio puede alentar la educación continua en el proceso de aprender a enseñar de por vida (Larsen-Freeman y Anderson, 2011). Para realizar la certificación a los estudiantes de Nivel Superior, se debe de tomar en cuenta aspectos relevantes sobre los diferentes métodos. Estos tienen la finalidad de hacer las clases más divertidas y dinámicas, además de hacer que el estudiante mejore en el idioma inglés.

\section{El Método de Traducción Gramatical} fue el primero en cuanto a la enseñanza del idioma inglés. Toma su nombre de la importancia que esta le da a la traducción de escritos y a la gramática (Larsen-Freeman y Anderson, 2011). Otro de los métodos utilizados es el Método Audiolingual. Este método supone que aprender un idioma se puede dividir en una serie de ejercicios donde se pone en práctica la repetición (Richards y Rodgers, 1999). Y por último (por mencionar algunos) tenemos el Enfoque Comunicativo, aunque no tiene el nombre de método lo encontramos como tal. Su objetivo es que los estudiantes desarrollen la habilidad de la comunicación.

\section{METODOLOGÍA}

La metodología del proyecto estuvo regida por los principios de la Investigación-Acción. El término puede considerarse como un amplio repertorio de definiciones y estrategias para la mejora del sistema social y educativo (Latorre, 2005). El siguiente proyecto siguió los principios básicos del modelo de Lewin retomados por Elliot (1993). Las fases propuestas por Elliott son: 1) Construcción del Plan de Acción (incluye revisar el problema inicial y las acciones concretas), 2) Visión de las formas de comenzar la siguiente acción y 3) Planificación de instrumentos para acceder a la información.

El proyecto se llevó a cabo en el Instituto de Educación Superior en Estudios de la Salud (IESALUD), ubicada en la Ciudad de Monterrey, Nuevo León. En el Instituto IESALUD se aplica el examen TOEFL PBT (Paper Based Test) a aquellos estudiantes que deseen presentarlo, es decir, no es de carácter obligatorio. Es por ello que la herramienta que se utilizó para realizar la propuesta fue a través de un ejemplar del TOEFL PBT para identificar el conocimiento del alumno en el idioma inglés, y con base a los resultados del examen se realizó una serie de actividades para potencializar la habilidad en la que más deficiencia tuvieron los estudiantes.

Los participantes en este proyecto fue un grupo de estudiantes que

\section{GRÁFICA 1. Resultados de la Puntuación (Score) del TOEFL PBT}

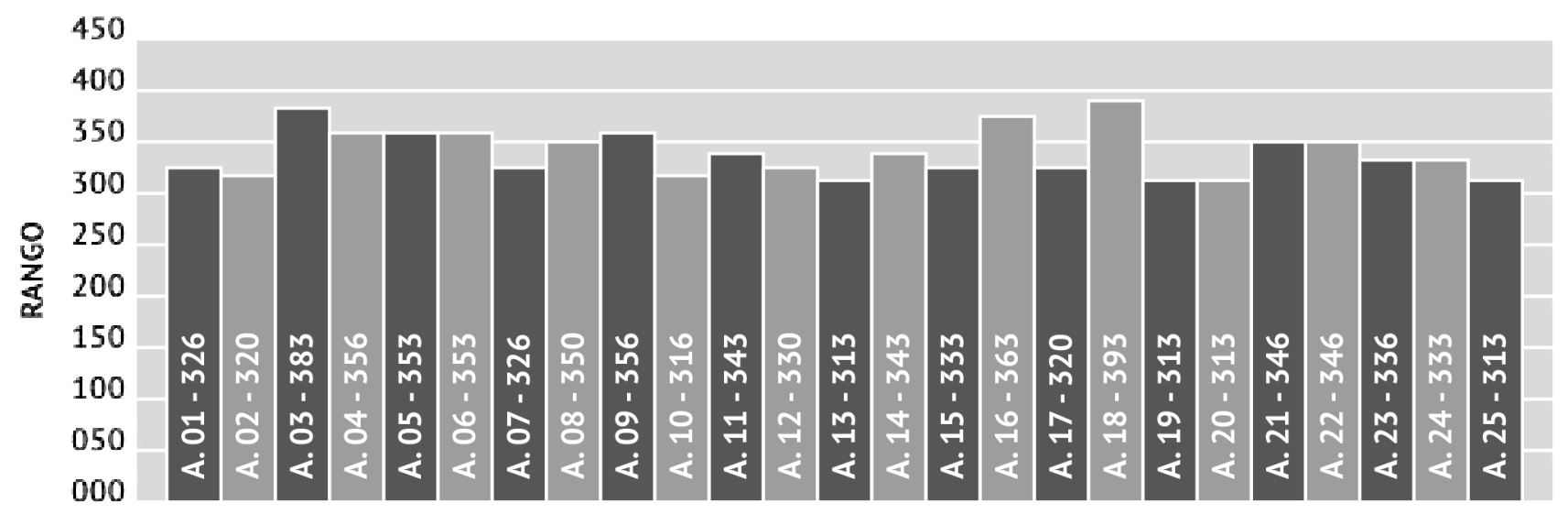

ALUMNOS 


\section{GRÁFICA 2.Promedio de Habilidades}

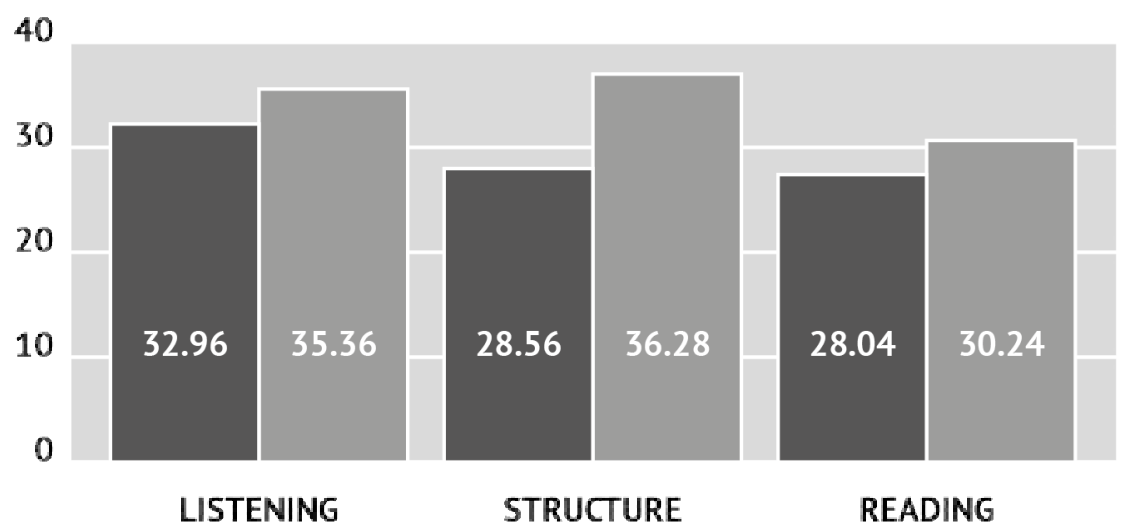

acreditó el inglés básico y llevan un nivel intermedio dando un total de 25 participantes de los cuales 19 son mujeres y 6 son hombres con edades que oscilan entre 19 y 30 años.

\section{RESULTADOS}

Se muestran las gráficas correspondientes a los resultados obtenidos por los 25 participantes, de los cuales se observan el puntaje (score) total del TOEFL PBT (Paper Based Test) y el promedio de cada una de las habilidades lingüísticas.

En la Gráfica 1 (pág. 50) se observa el puntaje (score) total que obtuvieron los estudiantes sumando las 3 habilidades (Listening, Structure y Reading) provenientes del ejemplar del TOEFL PBT. El puntaje total obtenido oscila entre 313 y 393 puntos. Esta puntuación ubica a los estudiantes de IESALUD en un nivel A1 del MCER. En la Gráfica 2 se realizó el promedio total de las 3 habilidades lingüísticas provenientes de los estudiantes que participaron en el proyecto de investigación. El promedio que se obtuvo para la habilidad del Listening fue de 32.96-35.36. Para la habilidad de Structure el promedio fue de 28.5636.28. Y por último, el promedio para la habilidad del Reading fue de 28.4- traducir los textos. Al concluir la propuesta se volvió a aplicar la prueba TOEFL PBT como prueba post-test.

En la Gráfica 3 se muestra que el promedio de la habilidad de la comprensión lectora (Reading) en el pre-test fue de 28.4-30.24. Al concluir las actividades brindadas en cada una de las sesiones y al realizar la prueba post-test nos percatamos que el promedio final de este fue de 29.8431.68, del pre-test al post-test hubo un incremento en la habilidad de la comprensión lectora (Reading) de $1.44 \%$, por lo que se logró aumentar esta habilidad.

Con respecto al resultado obtenido, se realizó una propuesta didáctica en la cual se implementaron actividades durante 3 semanas para reforzar e incrementar esta habilidad. El objetivo general de la propuesta fue incrementar la comprensión lectora (Reading)en los estudiantes con el fin de lograr una certificación del inglés como lengua extranjera a través de actividades que promuevan la comprensión lectora. El método que se utilizó para implementar las actividades fue el Método de Traducción Gramatical, dada la importancia a

\section{CONCLUSIÓN}

Hoy en día es muy importante contar con una certificación en el idioma inglés. Los alumnos juegan un papel importante ya que son estos mismos los que deben prepararse para demostrar su conocimiento en el idioma inglés. Los profesores deben darles las herramientas necesarias para potencializar sus habilidades, esto con la finalidad de prepararlos para obtener una certificación al mismo tiempo que los preparamos para su futuro en este mundo exigente en el que el certificado habla por sí solo.

\section{GRÁFICA 3. Promedio de Habilidad de la Comprensión Lectora}

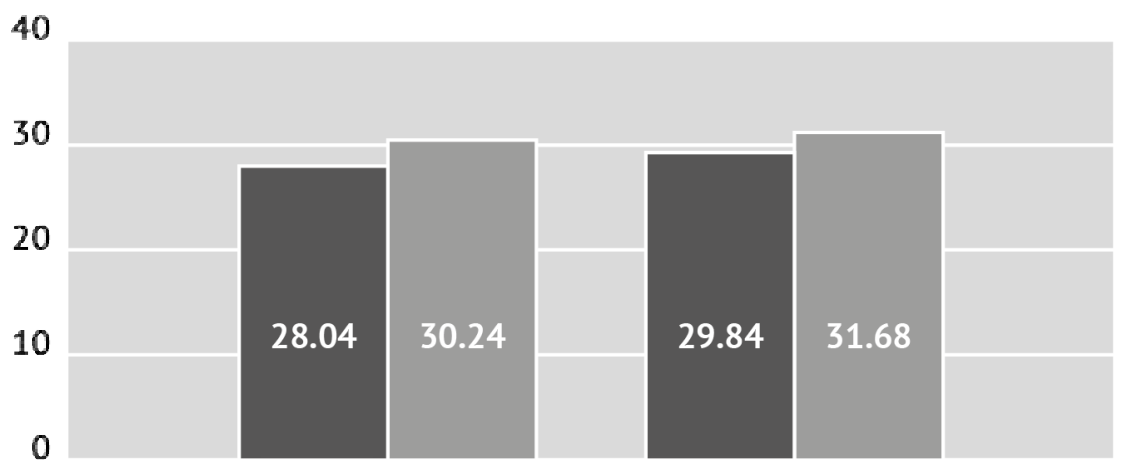

PRE-TEST
POST-TEST 


\section{REFERENCIAS}

Council of Europe. (2001). Marco común europeo de referencia para las lenguas: aprendizaje, enseñanza, evaluación. Recuperado de: https://cvc.cervantes.es/ensenanza/biblioteca_ele/ marco/cvc mer.pdf.

Education First (2017). Exámenes y Certificados de Inglés. Recuperado de: http://www. ef.com.mx/certificados-ingles.

Educational Testing Service (2017). Acerca del examen TOEFL PBT. Recuperado de: https:// www.ets.org/es/toefl/pbt/about.

Elliot, J. (1993). El cambio educativo desde la investigación-acción. Madrid: Morata.

Jitendra, A. K., \& Gajria, M. (2011). Reading comprehension instruction for students with learning disabilities. Focus on Exceptional Children, 43(8), 1-16. Recuperado de: http://www. highbeam.com/publications/focus-on-exceptional-childrenp106157.

Johnston, A. M., Barnes, M. A., \& Desrochers, A. (2008). Reading comprehension: Developmental processes, individual differences, and interventions. Canadian Psychology, 49(2), 125-132.

Larsen-Freeman, D. \& Anderson, M. (2011). Techniques and Principles in Language Teaching. New York: Oxford University Press.

Latorre,A. (2005). La investigación-acción conocer y cambiar la práctica educativa. Barcelona: Graó.

Organización Mexicanos Primero. (2015). Sorry el aprendizaje de inglés en México.De: http:// www.mexicanosprimero.org/index.php/educacion-en-mexico/como-esta-la-educacion/ estado-de-la-educacion-en-mexico/sorry-2015.

Real Academia Española. (2009). Diccionario de la lengua española. Recuperado de: https:// dle.rae.es/?id=A0fanvT|A0gTnnL.

Richards, J. \& Rodgers, T. (1999). Approaches and Methods in Language Teaching. New York: Cambridge University Press.

Ricoy, M. \& Álvarez, S. (2016). La enseñanza del inglés en la educación básica de personas jóvenes y adultas. Revista Mexicana de Investigación Educativa, 21(69), 385-409.
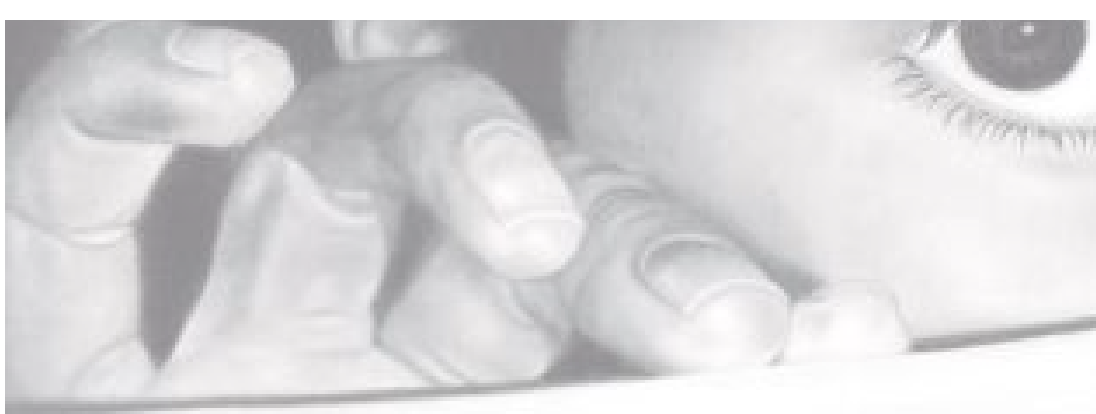
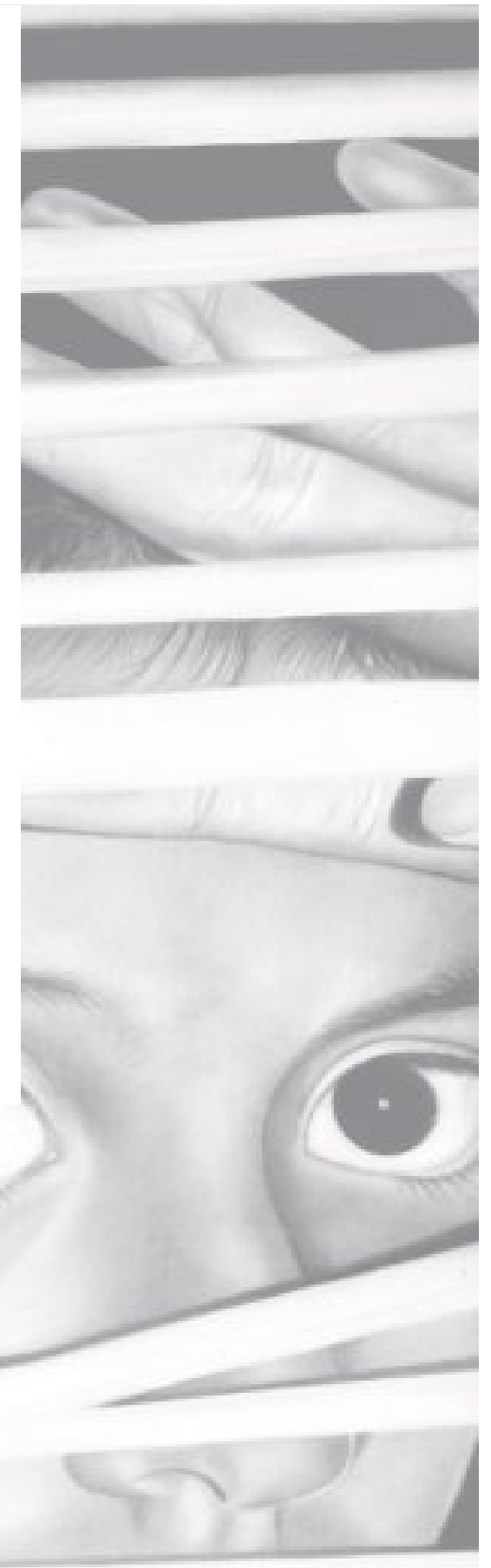

\section{Presencia Universitaria}




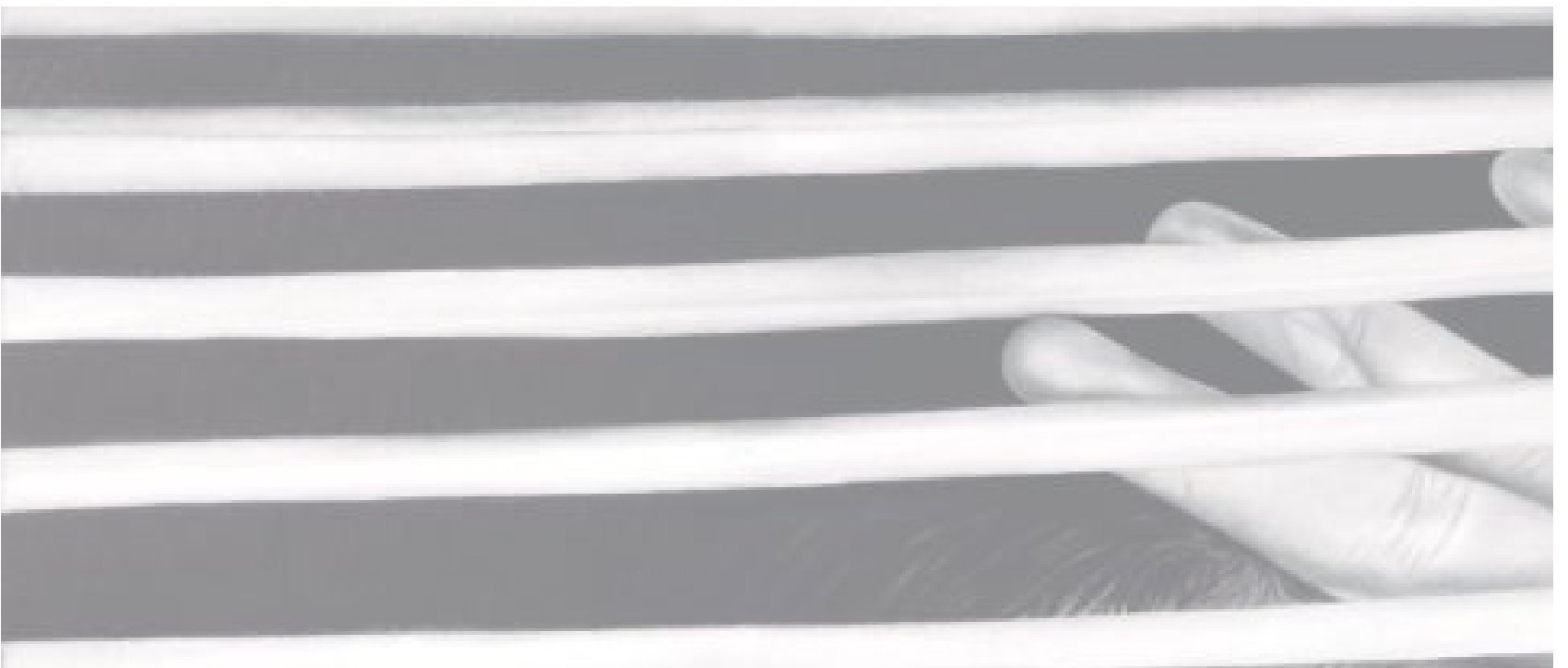

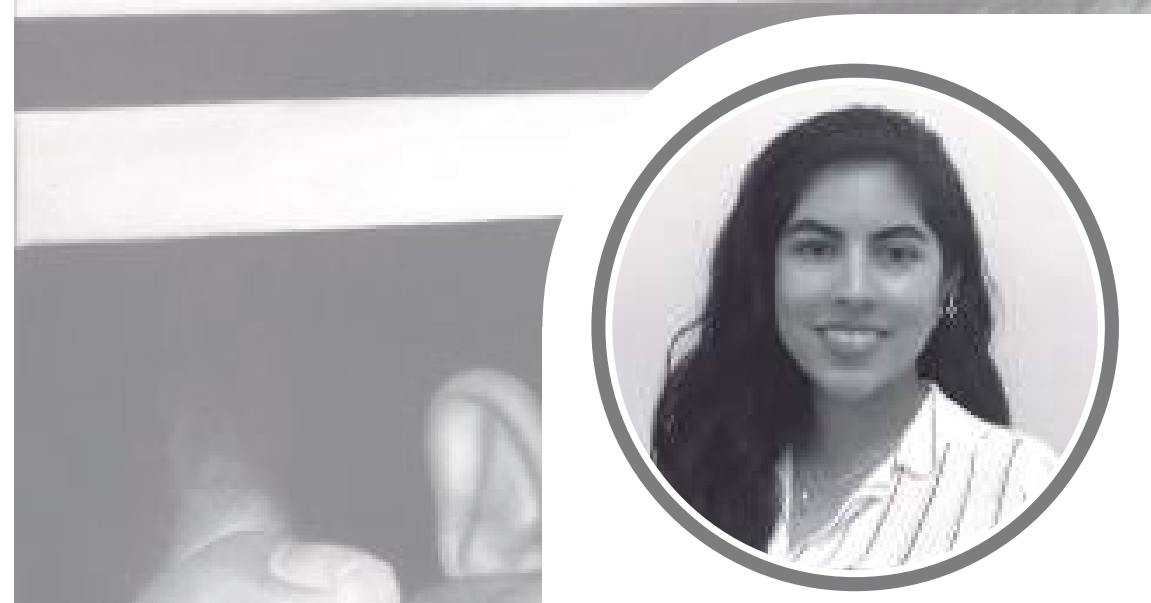

\section{Aglae Beatriz Flores Leos}

Es egresada de la Facultad de Enfermería de la Universidad Autónoma de Nuevo León. Ha realizado cursos de inglés y francés en diversas instituciones de idiomas del estado. Sus estudios de posgrado los realizó en la Facultad de Filosofía y Letras de la Universidad Autónoma de Nuevo León en la Maestría en Lingüística Aplicada en la Enseñanza de Lenguas Extranjeras.

\section{Correo Electrónico: agladeth@gmail.com}

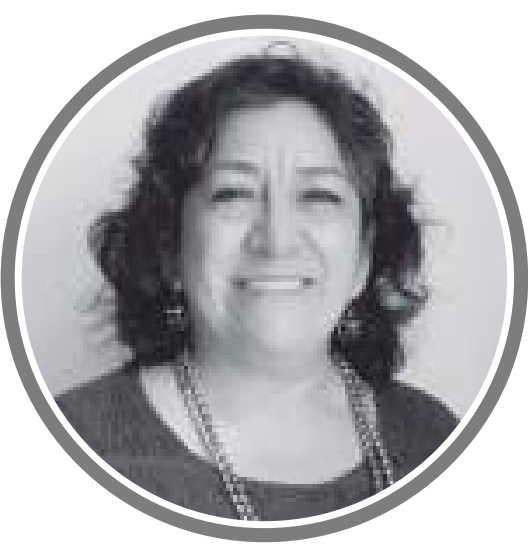

\section{María Guadalupe Rodríguez Bulnes}

Doctorado en Educación por The University of Birmingham en Inglaterra y Licenciada en Traducción de Inglés por la UANL. Sus proyectos de investigación y ponencias giran en torno de las temáticas: Formación Docente y Competencia Intercultural en la Enseñanza y Aprendizaje de Lenguas Extranjeras. Coordinadora de los procesos de acreditación para agencias nacionales e internacionales en la Dirección de Acreditación y Evaluación Internacional de la UANL.

Correo Electrónico:

lupira@hotmail.com

Recibido: 15/06/2019

Aceptado: 10/09/2019 